\title{
Performance Evaluation of Field-Enhanced P-Channel Split-Gate Flash Memory
}

\author{
Wen-Ting Chu, Hao-Hsiung Lin, Yu-Hsiung Wang, Chia-Ta Hsieh, Yung-Tao Lin, and Chung S. Wang
}

\begin{abstract}
A p-channel split-gate Flash memory cell, employing a field-enhanced structure, is investigated in this letter. A cell with a sharp poly-tip structure is utilized to enhance the electric field, while using Fowler-Nordheim tunneling through the interpoly oxide. The cell demonstrated an erase voltage as low as $12 \mathrm{~V}$. In cell programming, both channel-hot-hole impact ionization induced channel-hot-electrons (CHE) and band-to-band tunneling induced hot electrons (BBHE) are evaluated. BBHE shows an injection efficiency of $\sim 2$ orders in magnitude higher than that of CHE. The cell also demonstrated an acceptable program disturb window, which is of high concern in a p-channel stacked-gate cell. Both programming approaches can pass $300 \mathrm{k}$ program/erase cycles.
\end{abstract}

Index Terms-Field-enhanced structure, Flash memory, p-channel, split-gate.

\section{INTRODUCTION}

W HEN compared with its n-channel counterpart, p-channel Flash memory offers advantages in fast programming speed, low power consumption, high scalability, and is hot-hole-injection free [1], [2], [6]-[8]. The split-gate structure is designed to be immune to the "over-erase" issue present in n-channel cells [4], and it has the same immunity to the "over-program" issue in p-channel cells. However, the stacked-gate p-channel cell suffers from serious program disturb issues [8], which is usually solved by utilizing a 2-T approach. The split-gate cell has a built-in select-transistor, which can be used to reduce channel leakage so as to decrease the disturb probability, and requires less cell area. In this study, an inhibited source voltage is employed while using channel-hot-hole impact ionization induced channel-hot-electrons (CHE) in cell programming, which will increase the program disturb margin. On the other hand, band-to-band tunneling induced hot electrons (BBHE) also demonstrated a high immunity to program disturb. In addition, a sharp poly-edge structure can be utilized in a split-gate cell to enhance the electric field while the cell is being erased using Fowler-Nordheim $(\mathrm{F}-\mathrm{N})$ tunneling through the interpoly oxide, therefore, the erase voltage can be reduced [7]. Furthermore, there will also be no stress-induced leakage current (SILC) of the poly-to-poly field-enhanced F-N tunneling erase [11], [12], which is important for preventing any data retention problem. In this letter, both CHE and BBHE

Manuscript received June 8, 2005. The review of this letter was arranged by Editor A. Wang.

W.-T. Chu and H.-H. Lin are with the Department of Electrical Engineering and Graduate Institute of Electronics Engineering, National Taiwan University, Taipei, Taiwan, R.O.C.

Y.-H. Wang, C.-T. Hsieh, Y.-T. Lin, and C. S. Wang are with the Non-volatile Memory Division, Taiwan Semiconductor Manufacturing Company, Ltd., Hsinchu 300, Taiwan, R.O.C. (e-mail: wtchu@epicenter.ee.ntu.edu.tw).

Digital Object Identifier 10.1109/LED.2005.853633 (a)

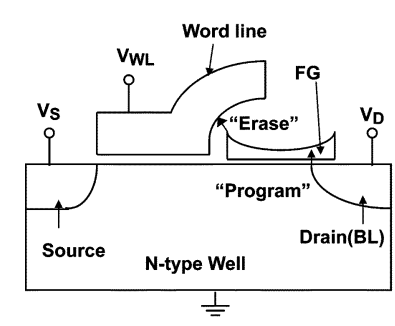

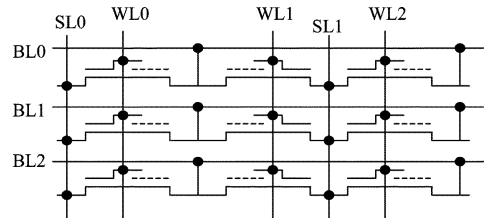

(b)

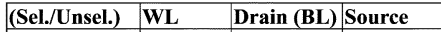
PGM(CHE) $-1.8 \mathrm{~V} / 0 \mathrm{~V}-10 \mathrm{~V} /$ Float $-0.5 \mathrm{~V} /-0.5 \mathrm{~V}$ PGM(BBHE) 8.5V/0V $-10 \mathrm{~V} / 0 \mathrm{~V}$ Float/Float \begin{tabular}{|l|l|l|l}
\hline Erase & $13 \mathrm{~V} / 0 \mathrm{~V}$ & $0 \mathrm{~V} / 0 \mathrm{~V}$ & $0 \mathrm{~V} / 0 \mathrm{~V}$ \\
\hline
\end{tabular}

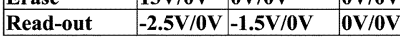

(c)
Fig. 1. (a) Cross section of the split-gate cell under study. (b) Memory array schematic diagram. (c) Typical cell operation conditions.

programming schemes are compared. BBHE shows an injection efficiency $\left(I_{\mathrm{FG}} / I_{D}\right)$ of $\sim$ two orders of magnitude higher than that of CHE. Moreover, BBHE also has minor gate current degradation after stress than CHE. Both programming schemes have passed $300 \mathrm{k}$ program/erase $(\mathrm{P} / \mathrm{E})$ cycles.

\section{DEVICE FABRICATION}

Double polysilicon $0.18 \mu \mathrm{m}$ CMOS technology was used to fabricate a split-gate cell, $0.65 \mu \mathrm{m}^{2}$ in area, with a sharp floating-gate (FG) edge, as shown in Fig. 1(a) [3], [5], [10]. After shallow trench isolation and $\mathrm{N}$-well formation, the first gate oxide, i.e., FG oxide, was grown on the Si-substrate, then the poly and SiN layers were deposited sequentially using LPCVD. The FG was defined using photolithography, and the $\mathrm{SiN}$ layer was removed using dry etching to expose the FG area. Next, polysilicon oxidation was performed to generate a bowl-shaped polystructure. After removing the residual SiN, the sharp poly-edge was completed by performing a polyetch using poly-oxide as a hard mask. Interpoly-oxide was then deposited to form both the tunnel oxide and the word line (WL) oxide. Finally, the second polysilicon (as WL) was deposited and patterned. The memory array schematic diagram is shown in Fig. 1(b).

\section{RESULTS AND DISCUSSION}

Typical p-channel split-gate cell operations are shown in Fig. 1(c). While the cell is being erased, the voltage difference between the WL and the other nodes can be as low as $12 \mathrm{~V}$ (see Fig. 2) as a result of the introduction of a field-enhanced structure. While programming the cell using CHE, the WL and drain bias are -1.8 and $-10 \mathrm{~V}$, respectively, and the source voltage is $-0.5 \mathrm{~V}$, which is designed to reduce channel leakage so as to improve the disturb margin. As shown in Fig. 3(a), 


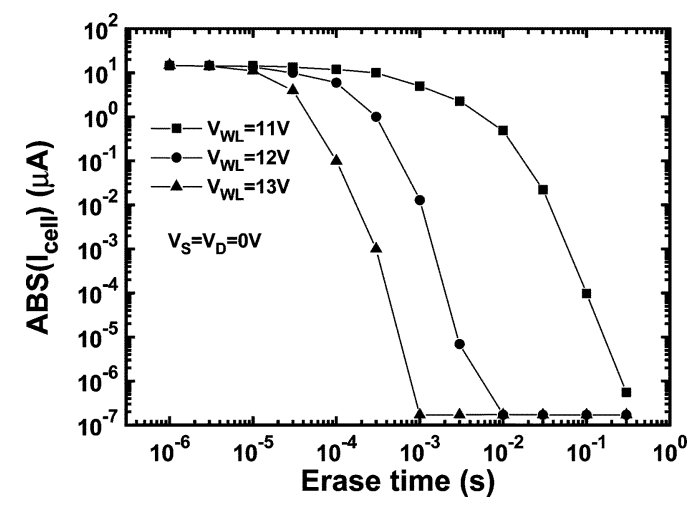

Fig. 2. $I_{\text {cell }}$ versus erase time for various WL voltages. $\left[I_{\text {cell }}\right.$ denotes the read-out current of the cell with the condition shown in Fig. 1(c)].

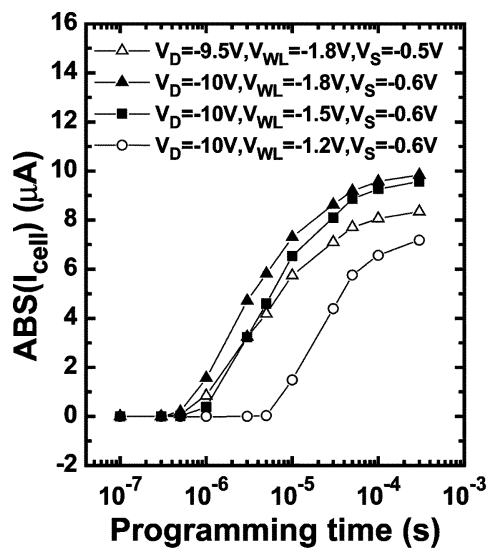

(a)

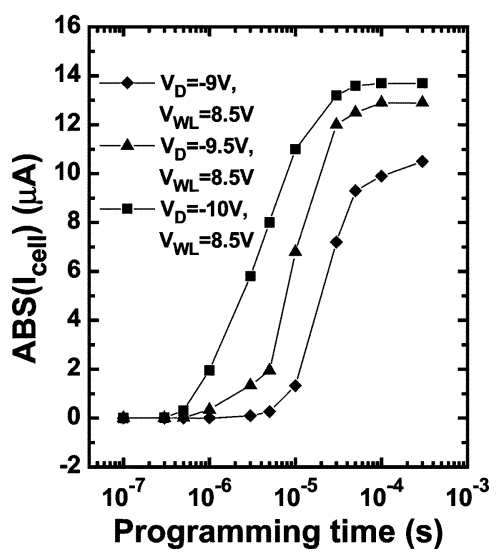

(b)

Fig. 3. $I_{\text {cell }}$ versus programming time for (a) CHE (b) BBHE approaches.

the cell can be well-programmed within tens of microseconds. While programming the cell using BBHE, the WL and drain bias are 8.5 and $-10 \mathrm{~V}$, respectively. As shown in Fig. 3(b), the cell can be well-programmed within $10 \mu$ s. A cell programmed using BBHE has a higher cell current than that using CHE, since BBHE uses $8.5 \mathrm{~V}$ on the $\mathrm{WL}$, which will couple the FG to a higher potential and attract more electrons to be injected into the FG. As shown in Fig. 4(a), while programming the cell using CHE, $\underline{V}_{\mathrm{S}}=0 \mathrm{~V}$ has a more serious program disturb than other $\underline{V}_{\mathrm{S}}$ values due to a higher channel leakage. Programming using $\mathrm{CHE}$ demonstrates that the cell current is still lower than $1 \mu \mathrm{A}$ after $30 \mathrm{k}$ program pulses (10 $\mu$ s per pulse) when the $\left|V_{\mathrm{S}}\right|$

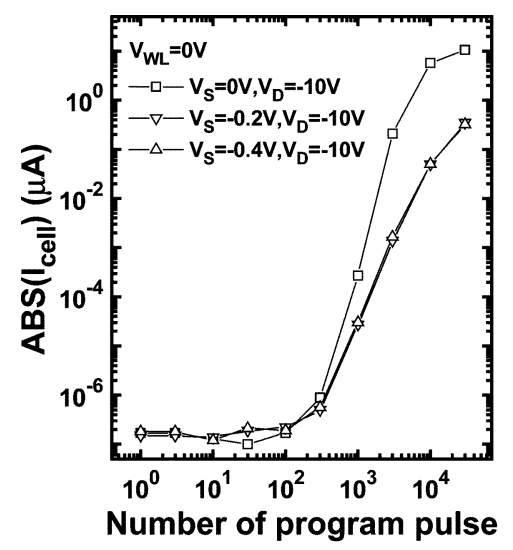

(a)

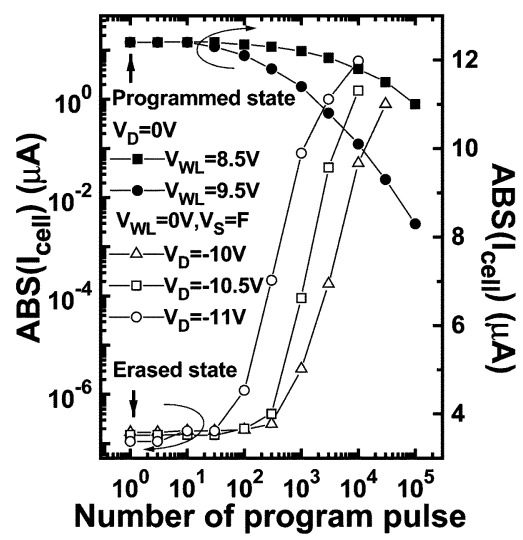

(b)

Fig. 4. Program disturb window check for (a) CHE (b) BBHE (including the programmed and erased states) approaches.

is higher than $0.2 \mathrm{~V}$. Fig. 4(b) shows the program disturb in both the programmed and erased state when programming the cell using BBHE. The BBHE program disturb on the erased state is for the unselected cell in the same BL. It was found that the higher the $V_{D}$, the faster the cell current increase becomes. The cell current is still lower than $1 \mu \mathrm{A}$ after $30 \mathrm{k}$ program pulses ( $10 \mu$ s per pulse) when $V_{D}=-10 \mathrm{~V}$. On the other hand, the BBHE program disturb on the cell in the programmed state in the same WL also shows acceptable immunity to program disturb. The drop in cell current is less than $20 \%$ after $30 \mathrm{k}$ program pulses when $V_{\mathrm{WL}}=9.5 \mathrm{~V}$. The number of program pulses required for the program disturb in real applications should be in the thousands.

In addition, as shown in Fig. 5, BBHE shows an injection efficiency of $\sim 2$ orders in magnitude higher than that of CHE. However, CHE in a p-channel split-gate cell does not have the same high injection efficiency as its n-channel counterpart using source-side injection. Programming using BBHE also shows a minor injection efficiency degradation when compared to CHE. Fig. 6 shows that the cells have passed $300 \mathrm{k} \mathrm{P} / \mathrm{E}$ cycles for both programming approaches. However, it can be seen that after $100 \mathrm{k}$ cycles, the erased-state cell current increases because of: 1) a degradation in the $\mathrm{F}-\mathrm{N}$ tunneling ability owing to the interpoly oxide electron trapping during cell erasing and 2) the reduction in the magnitude of the FG threshold voltage, resulting from the FG oxide trapped charges during cell programming [6], 


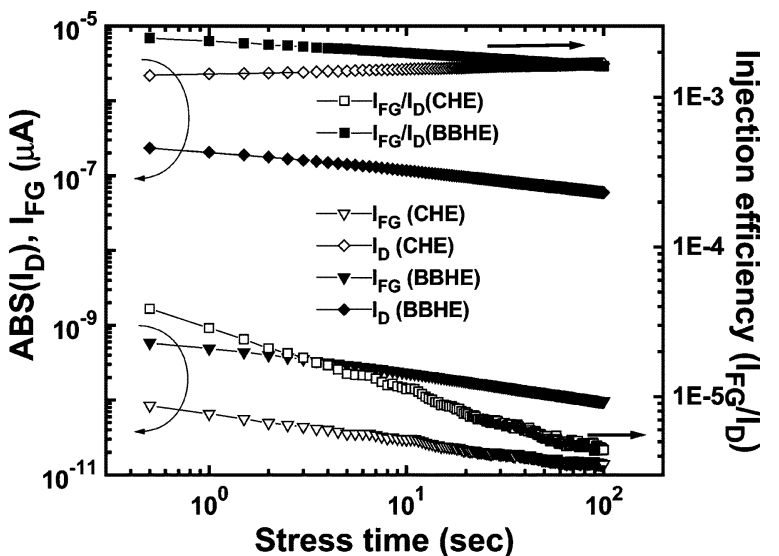

Fig. 5. FG-connected cell is used to measure the FG current. BBHE shows an injection efficiency of $\sim$ two orders of magnitude higher than that of CHE. BBHE also shows minor injection efficiency degradation than that of CHE. The conditions for CHE are: $V_{D}=-9 \mathrm{~V}, V_{\mathrm{FG}}=-2 \mathrm{~V}, V_{S}=-0.5 \mathrm{~V}$, $V_{\mathrm{WL}}=-1.8 \mathrm{~V}$; for BBHE are: $V_{D}=-9 \mathrm{~V}, V_{\mathrm{FG}}=0 \mathrm{~V}, V_{S}=$ floating.

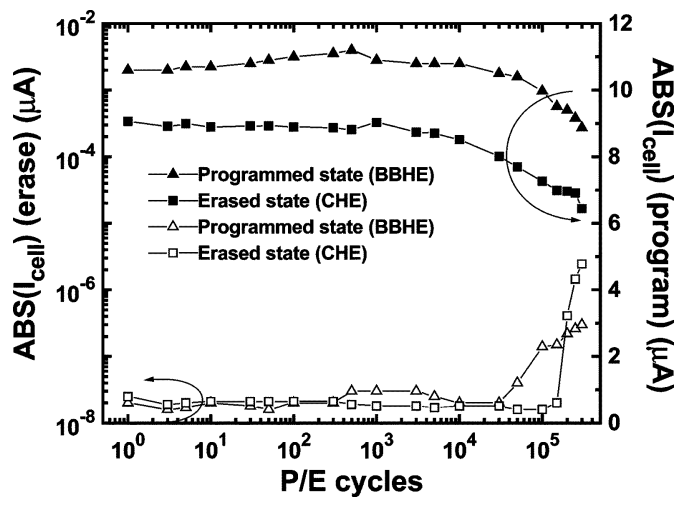

Fig. 6. Cell can pass the P/E cycling endurance test $300 \mathrm{k}$ times. However, it can be seen that after $100 \mathrm{k}$ cycles, the erased-state cell current increases. The conditions for CHE programming are: $V_{D}=-10 \mathrm{~V}, V_{S}=-0.5 \mathrm{~V}$, $V_{\mathrm{WL}}=-1.8 \mathrm{~V}, 10 \mu \mathrm{s}$; for BBHE programming are: $V_{D}=-10 \mathrm{~V}, V_{S}=$ floating, $V_{\mathrm{WL}}=8.5 \mathrm{~V}, 10 \mu \mathrm{s}$; for $\mathrm{F}-\mathrm{N}$ tunneling erase are: $V_{\mathrm{WL}}=13 \mathrm{~V}$, $V_{D}=0 \mathrm{~V}, V_{S}=0 \mathrm{~V}, 10 \mathrm{~ms}$.

[9]. The FG oxide trapped charges effect also retards the ability of the electron to be injected into the FG and results in a drop in the programmed-state cell current.

\section{CONCLUSION}

In this letter, a p-channel split-gate Flash memory cell with a field-enhanced structure has been used to demonstrate a low erase voltage of $12 \mathrm{~V}$ using poly-poly $\mathrm{F}-\mathrm{N}$ tunneling, with a high immunity to program disturb and the ability to pass $300 \mathrm{k} \mathrm{P} / \mathrm{E}$ cycles, while being programmed using either CHE or BBHE. However, BBHE has an injection efficiency of $\sim 2$ orders in magnitude higher than that of CHE, so BBHE is highly suitable for low power applications. The process in this paper is compatible with that of standard logic, so it is a very promising cell for use in both commodity and embedded applications.

\section{ACKNOWLEDGMENT}

The authors would like to thank the members of the Nonvolatile Memory Program, R\&D Division, Taiwan Semiconductor Manufacturing Co. Ltd., for wafer fabrication and Silicon Storage Technology, Inc., Sunnyvale, CA, for technical support.

\section{REFERENCES}

[1] T. Ohnakado, K. Mitsunaga, M. Nunoshita, H. Onoda, K. Sakakibara, N. Tsuji, N. Ajika, M. Hatanaka, and H. Miyoshi, "Novel electron injection method using band-to-band tunneling induced hot electron (BBHE) for Flash memory with a P-channel cell," in IEDM Tech. Dig., 1995, pp. 279-282.

[2] C. C.-H. Hsu, A. Acovic, L. Dori, B. Wu, T. Lii, D. Quinlan, D. DiMaria, Y. Taur, M. Wordeman, and T. Ning, "A high speed, low power P-channel Flash EEPROM using silicon rich oxide as tunneling dielectric," Ex. Abst. SSDM, p. 140, 1992.

[3] B. Yeh, "Single Transistor Non-Volatile Electrically Alterable Semiconductor Memory Device," U.S. Patent 5029 130, 1991.

[4] T. C. Ong, A. Fazio, N. Mielke, S. Pan, N. Righos, G. Atwood, and S. Lai, "Erratic erase in ETOX Flash memory array," in VLSI Symp. Tech. Dig., 1993, pp. 83-84.

[5] S. Kianian, A. Levi, D. Lee, and Y.-W. Hu, "A novel 3 volts-only, small sector erase, high density Flash E2PROM," in Symp. VLSI Tech. Dig., 1994, pp. 71-72.

[6] S. S. Chung, S. N. Kuo, C. M. Yih, and T. S. Chao, "Performance and reliability evaluations of P-channel Flash memories with different programming schemes," in IEDM Tech. Dig., 1997, pp. 295-298.

[7] T. Ohnakado, H. Onoda, O. Sakamoto, K. Hayashi, N. Nishioka, H. Takada, K. Sugahara, N. Ajika, and S. Satoh, "Device characteristics of $0.35 \mu \mathrm{m}$ p-channel DINOR Flash memory using band-to-band tunneling-induced hot electrons (BBHE) programming," IEEE Trans. Electron Devices, vol. 46, no. 11, pp. 1866-1871, Nov. 1999.

[8] F. R.-L. Lin and C. C.-H. Hsu, "New divided-source structure to eliminate instability of threshold voltage in P-channel Flash memory using channel hot-hole-induced-hot-electron programming," in Symp. VLSI Tech. Dig., 1999, pp. 203-206.

[9] Y.-L. Chu and C.-Y. Wu, "A new observation of band-to-band tunneling induced hot carrier stress using charge-pumping technique," IEEE Electron Device Lett., vol. 21, no. 3, pp. 123-126, Mar. 2000.

[10] W.-T. Chu, H.-H. Lin, Y.-L. Tu, Y.-H. Wang, C.-T. Hsieh, H.-C. Sung, Y.-T. Lin, C.-S. Tsai, and C. S. Wang, "Using an ammonia treatment to improve the floating-gate spacing in split-gate Flash memory," IEEE Electron Device Lett., vol. 25, no. 9, pp. 616-618, Sep. 2004.

[11] W. D. Brown and J. E. Brewer, "Floating gate nonplanar devices," in Nonvolatile Semiconductor Memory Technology, 1st ed. Piscataway, NJ: IEEE Press, 1987, ch. 3.

[12] W.-T. Chu, H.-H. Lin, Y.-H. Wang, C.-T. Hsieh, H.-C. Sung, Y.-T. Lin, and C. S. Wang, "High SCR design for one-transistor split-gate full-featured EEPROM," IEEE Electron Device Lett., vol. 25, no. 7, pp. 498-500, Jul. 2004 